\title{
Prioritäre Themen in der Versorgungsforschung zur psychischen Gesundheit
}

\author{
Priorities in Mental Health Services Research
}

Autoren

Institute
Anke Bramesfeld ${ }^{1}$, Steffi Riedel-Heller ${ }^{2}$

Forschungsnetz psychische Gesundheit, Zentrum für Psychische Gesundheit, Klinik und Poliklinik für Psychiatrie, Universität Leipzig

Professur für Public Health, Zentrum für Psychische Gesundheit, Klinik und Poliklinik für Psychiatrie, Universität Leipzig
Bibliografie

DOI $10.1055 / \mathrm{s}-0028-1090027$

Psychiat Prax 2008; 35 :

315-317 @ Georg Thieme

Verlag KG Stuttgart · New York .

ISSN 0303-4259

Korrespondenzadresse

Dr. Anke Bramesfeld, MPH

Leiterin Forschungsnetz psy-

chische Gesundheit, Klinik

und Poliklinik für Psychiatrie,

Universität Leipzig

Semmelweisstraße 10

04103 Leipzig

Anke.bramesfeld@medizin.

uni-leipzig.de
Versorgungsforschung liefert die wissenschaftliche Grundlage, ,um die Akteure im Gesundheitswesen, insbesondere die Politik auf der Basis valider wissenschaftlicher Erkenntnisse in größtmöglicher Objektivität und Transparenz zu unterstützen und zu beraten " [1]. Diese Definition der Bundesärztekammer trifft nicht nur für die Versorgungsforschung allgemein zu, sondern beschreibt auch treffend den Anspruch der Versorgungsforschung im Bereich der psychischen Gesundheit.

Unter Berücksichtigung der Begrenztheit finanzieller als auch personeller Ressourcen in der Versorgungsforschung ist es sinnvoll, gezielt Prioritäten zu setzen. Prioritäten in der Allokation von Versorgungs- und Forschungsressourcen wurden wiederholt kritisiert, da sie der Bedürftigkeit oder Relevanz bestimmter Versorgungsgebiete nicht entsprächen [2,3]. Häufig konzentrieren sich Ressourcen in Bereichen, auf die sich die gesellschaftliche und politische Aufmerksamkeit konzentriert, sowie auf Bereiche, die den Forschern (meist universitätsgebundene krankenhaussozialisierte Wissenschaftler) leicht zugänglich sind. Darüber hinaus nehmen Interessengruppen Einfluss auf die Prioritätensetzung, wie z. B. Industrie, Verbände und Versorgungsanbieter [4]. D.h. Versorgungsforschung agiert immer im Spannungsfeld von Interessen der beteiligten Akteure (Wissenschaftler, Versorgungsanbieter, Finanziers, Entscheidungsträger, Betroffene).

Was im Zusammenhang mit Versorgung und Versorgungsforschung als Priorität angesehen wird, ist abhängig von den Experten und Akteuren, die konsultiert werden [5]. Je nach Hintergrund werden Prioritäten unterschiedlich ausgedrückt: Manager drücken Prioritäten in Form von Diskussionspunkten aus, politische Akteure in Form von Interessengruppen, die Öffentlichkeit definiert Prioritäten anhand von Problemen, Wissenschaftler anhand von Disziplinen oder
Methoden und Klinker sehen vor allem Krankheiten [6]. Darüber hinaus spielen ökonomische Bewertungen in der gesundheitspolitischen Diskussion um Prioritäten eine zunehmend wichtige Rolle [5]. Angesichts der Unterschiede im Zugang zu Prioritäten gestaltet sich der Prozess der Prioritätenfindung in Versorgung und Versorgungsforschung weniger technisch als interpretativ und in Form eines Diskurses. Von erheblicher Bedeutung ist das frühzeitige Einbinden von Entscheidungsträgern. Effektive Interaktion zwischen Wissenschaftlern, Entscheidungsträgern, Versorgungsnutzern und Finanziers beim Identifizieren und Formulieren von Forschungsfragen erhöht die Wahrscheinlichkeit, dass die Forschungsergebnisse später tatsächlich in der Versorgung umgesetzt werden [6].

Was aber sind gegenwärtig prioritäre Themen der Versorgungsforschung im Bereich psychische Gesundheit in Deutschland? Diese Frage diskutierten im April 2008 einen Tag lang 30 Wissenschaftler und Akteure aus der Versorgung psychisch kranker Menschen. Es waren hierzu sowohl Kliniker als auch reine Versorgungsforscher, Vertreter der beteiligten Ministerien, Akteure aus den Verbänden und der Industrie sowie Betroffenen-Vertreter eingeladen worden. Die Diskussion orientierte sich an Impulsreferaten, die das breite Spektrum der relevanten Themenbereiche umreißen sollten: Epidemiologie, medizinische Versorgungssituation, Versorgungssituation im komplementären Bereich, Prävention, gesundheitspolitische Entwicklung und regionale Vergleiche in der Versorgungsforschung. Das Ergebnis war eine Liste mit 20 Themen aus der Versorgungsforschung im Bereich psychische Gesundheit. Diese Liste wurde in einem Priorisierungsprozess an alle Beteiligte versandt und jeder Experte kennzeichnete die 7 ihm am wichtigsten erscheinenden Themen. Diese wurden dann durch Zuweisung von Punkten - 7 für das wichtigste, einer für das siebtwichtigste Thema 
- hierarchisiert. Von 28 angeschriebenen Experten antworteten 24, 21 Antworten gingen in die Auswertung ein. Die Antwortenden waren überwiegend Wissenschaftler der Versorgungsforschung, 9 gaben an auch aktiv in der Versorgung tätig zu sein, 3 in der Administration und einer war ein Vertreter der Industrie. Die auf diese Art und Weise identifizierten 7 wichtigsten Themen werden im Folgenden vorgestellt und diskutiert.

Thema 1: Versorgungswege: Anreizanalyse: Wer entscheidet wie wann er wo medizinische Hilfe in Anspruch nimmt, wo sind Stellen an denen Personen „im Versorgungssystem gefiltert“ werden? Dieses Thema erhielt 63 Punkte, 15 Experten hatten dieses Thema als eines unter den 7 wichtigsten genannt, allerdings hatte nur einer von ihnen es auch als „das Wichtigste“ bezeichnet.

Hintergrund ist die inzwischen sprichwörtliche Unterversorgung psychisch kranker Menschen, wie sie immer wieder in den verschiedensten Studien nachgewiesen wird [7-9]. Hieraus ergibt sich die Frage, an welchen Stellen im Versorgungssystem Personen, die Hilfe bedürften, aus dem System fallen, aber auch wo die Eintrittspunkte in das System sind und welche Motivationen der Patienten beim Eintritt in das System eine Rolle spielen.

- Thema 2: Präventionsforschung: longitudinaler Ansatz, Kohortenstudien, Stepped-Care-Ansätze unter Berücksichtigung von Kosteneffektivität und verschiedenen Outcomes (Gewalt, Heimeinweisung, Chronifizierung); sowie unter Berücksichtigung von potenziellen Modifikatoren und Hintergrundvariablen (Lebensstil, kulturelle Strömungen, Religiosität ). Dieses Thema erhielt insgesamt 58 Punkte von 13 Experten, von denen 5 es als das siebtwichtigste Thema benannten.

Hintergrund: Für die Effektivität von Präventionsmaßnahmen im Bereich psychische Gesundheit liegt einige Evidenz vor [10]. Gleichzeitig erweisen sich Interventionen, in die große Hoffnungen gesetzt wurden und die weit verbreitet sind als wenig bis gar nicht effektiv. So sind z. B. die Ergebnisse der Suchtprävention bei Kindern und Jugendlichen höchst inkonsistent [11-15]. Nach Aussage der Experten würden zu wenig kontextuelle Bedingungen und Subgruppen bei der Erarbeitung und der Evaluation von Prävention berücksichtigt. Langzeitstudien liegen fast gar keine vor. Auch über die Kosteneffektivität präventiver Maßnahmen ist wenig bekannt. Gesucht werden kosteneffektive Interventionen, die auch nachhaltig longitudinal den Lebensstil beeinflussen und zu einer stärkeren Resilienz führen.

- Thema 3: Ressourcenallokation (stationär vs. ambulant; leichte vs. schwere Störungen): 57 Punkte wurden von 11 Experten vergeben.

Hintergrund: Die meisten psychischen Erkrankungen werden ambulant behandelt, die meisten Ressourcen werden aber für die stationäre Behandlung aufgebracht [16]. Während Behandlungsstrukturen für leichtere und mittlere Störungen ausgebaut werden, sind Strukturen für die sozialpsychiatrische Versorgung in einigen Regionen rar. Mehr Wissen über die Verteilungsmechanismen im System ist nötig. Es werden Interventionsmöglichkeiten gesucht die erlauben, die Allokation gezielter im Hinblick auf den Bedarf von Schwerkranken zu steuern.

- Thema 4: Barrieren für eine optimierte Versorgung: Analyse von Barrieren aufseiten der Leistungserbringer und aufseiten der Nutzer: 45 Punkte von 13 Experten.

Hintergrund: Depressive Patienten und Angstpatienten erhalten eine unzureichende Versorgung [17]. Die ESEMeD-Studie erbrachte, dass in der hausärztlichen Versorgung die Chancen eine adäquate Depressionstherapie zu erhalten noch geringer sind als in der fachärztlichen Behandlung [18]. Was sind Ursachen? Welche Bedingungen beeinflussen die Adherenz von Patienten an eine Therapie? Welche Mechanismen und Anreize beeinflussen das Vorhandensein von bestimmten Versorgungsangeboten? Was bewegt z. B. einen Nervenarzt dazu seine Praxis mehr psychotherapeutisch oder mehr sozialpsychiatrisch auszurichten? Was sind Barrieren aufseiten der potenziellen Nutzer? Welche Einstellungen verhindern, dass sie Hilfe suchen? Die ESEMeD-Studie konnte zeigen, dass die Wahrscheinlichkeit professionelle Hilfe in Anspruch zu nehmen von Patientenmerkmalen wie Alter, Geschlecht, Bildung abhängt $[19,20]$.

- Thema 5: Komorbidität: Erforschen des Einflusses von psychischer Komorbidität auf den Verlauf und Therapieeffekte von somatischen Erkrankungen (43 Punkte von 10 Experten). Hintergrund: Psychische Erkrankungen beeinflussen den Outcome von somatischen Erkrankungen, insbesondere HerzKreislauf-Erkrankungen massiv [21-23]. Psychische Erkrankungen müssen als regelmäßige Komplikation bei der Behandlung von somatischen Erkrankungen berücksichtigt werden. Was aber bedeutet dies für Therapieempfehlungen in Leitlinien? Wie verändert sich Therapie und Prognose einer somatischen Erkrankung bei psychischer Komorbidität?

- Thema 6: Nutzen von Stepped-Care-Ansätzen, systematische Integration von Selbstmanagementelementen in die Regelversorgung (unter Nutzen neuer Medien) (43 Punkte von 11 Experten). Hintergrund: Eine inadäquate Patientenschulung insbesondere was die Befähigung zur aktiven Teilnahme an der Behandlung betrifft, beklagte bereits der Sachverständigenrat für die konzertierte Aktion im Gesundheitswesen im Jahr 2001 [24]. Systematische Befähigung von Patienten zum Selbstmanagement wurde von allen Teilnehmern des Think Tanks als unerlässlich angesehen. Hinsichtlich einer systematischen Hierarchisierung der Interventionen im Sinne von Stepped Care müssten je nach Schweregrad der Erkrankungen nicht alle Interventionen facharztbasiert durchgeführt werden. Gute Erfahrungen werden im Ausland auch mit internetbasierten Interventionen bei Depressions- und Angsterkrankungen gemacht.

Als siebtwichtig wurden 2 Themen bewertet. Beide erhielten 39 Punkte von jeweils 8 Experten:

- Thema 7 a) Monitoring von Gesetzen.

Hintergrund: Dies ist der klassische Ansatz des Health Impact Assessments (vgl. [25]): Welchen Einfluss haben strukturelle Veränderungen auf die Bevölkerungsgesundheit oder (als Proxy) auf die Versorgungssituation [26]? Zu denken ist z. B. an mögliche Auswirkungen von Budgetregulierungsmaßnahmen wie z. B. das Einführen von Leitsubstanzen zu denen auch SSRI zählten, auf die Versorgung depressiver Menschen. Andere Themen wären die Einführung von Zuzahlungen, oder aber ein neuer EBM und die Auswirkungen auf die Versorgung psychisch kranker Menschen (ändert sich z. B. die durchschnittliche Gesprächsdauer mit Patienten, wenn dies besser vergütet wird?). Gesundheitsökonomische Modellrechnungen können hilfreich sein beim Abschätzen der Folgen und der Sinnhaftigkeit von Reformen/Interventionen [27].

- Thema 7b) Benchmarking von Regionen: Identifizieren von Indikatoren für Qualität von Versorgungsangeboten, -systemen, -strukturen, -regionen.

Hintergrund: Die Versorgungslandschaft in Deutschland ist in vieler Hinsicht heterogen [28]. Dies betrifft unter anderem die Anzahl von Krankenhausbetten und niedergelassene Nervenärzten pro 100000 Einwohner, das Vorhandensein ambulanter psychiatrischer Pflege, das Vorhandensein von Rehabilitationsmaßnahmen [29]. Aber was bedeutet das? Ist dies Ausdruck von ungleicher Versorgung oder von individuell angepassten Lösungen für lokale Probleme? Was sind Parameter für eine gute/ adäquate Versorgung in einer Region? Psychiatrieplanung liegt in der Zuständigkeit der Länder und Regionen. Gute psychiatrische Versorgung geht über die Institutionen hinaus und erzielt nur im Verbund der Angebote ihre volle Wirkung. Daher hat das Gesamtpaket von Versorgung in einer Region und seine Kosteneffizienz über Schnittstellen hinaus große Relevanz.

Was ist prioritär? Themen die von besonders vielen Wissenschaftlern als „das Wichtigste“ gekennzeichnet wurden, oder Themen, die von vielen Wissenschaftlern als überhaupt wichtig genannt wurden? Waren alle Akteure im Priorisierungsprozess gleichermaßen eingebunden? Es gab mehr Vertreter der Wissenschaft und Klinik als der Verwaltung. Betroffenenvertreter waren zwar eingeladen, aber leider nicht gekommen. Hat dies den Priorisierungsprozess verzerrt? Auf diese Fragen wird es kei- 
ne eindeutigen Antworten geben, sowie es auch nie die eindeutige ultimative Prioritätenliste geben wird, sondern immer nur Themen, die in der Diskussion und Reflexion über Versorgung und Versorgungsforschung vorne stehen. Prioritäten können daher auch nicht nach dem Mehrheitsprinzip identifiziert werden, sondern immer nur im lebendigen, multidisziplinären Diskurs. Ein solcher Diskurs ist kennzeichnend für die Entscheidungsfindung in der Gesundheitspolitik von demokratischen Wohlfahrtsstaaten [30].

Die Ergebnisse des Think Tanks bieten ein eher beschreibendes Bild über Lücken und drängende Fragen in der Versorgungsforschung psychischer Gesundheit aus Sicht der mit Forschung in diesem Bereich befassten Akteure. Die überwiegende Anzahl der Themen, die als prioritär identifiziert wurden, drehen sich um Fragen auf Gesundheitssystemebene. Ein Bedarf nach mehr gesundheitssystemorientierter Forschung im Bereich psychischer Gesundheit wurde auch schon von anderen Stellen postuliert [31]. Klassische Evaluation von Wirksamkeit von Interventionen wird überwiegend für die relativ junge Disziplin der Prävention psychischer Krankheiten gefordert. Auf die Krankheitsversorgung und die Rehabilitation bezogen aber bedeutet das Ergebnis des Think Tanks: Es ist eigentlich bekannt was wirkt. Unbekannt ist wie diese Erkenntnisse am Besten umgesetzt werden und ob und wie erreicht werden kann, dass die Hilfe diejenigen erreicht, die sie am nötigsten brauchen.

Es bleibt zu wünschen, dass künftige Forschungsvorhaben, Forschungsausschreibungen und -förderungen die in diesem Think Tank angesprochenen prioritären Themen der Versorgungsforschung im Bereich psychischer Gesundheit berücksichtigen.

\section{Danksagung}

Wir danken allen Beteiligten des Think Tanks für eine von großer Expertise und Reflexion gekennzeichneten Diskussion. Unser Dank gilt: T. Becker, Psychiatrie Günzburg, Universität Ulm; F. Böcker, Saale-Unstrut-Klinikum Naumburg; C. Daiminger, Universität Magdeburg; A. Domdey, Firma Lundbeck; H. Elgeti, Psychiatrie Medizinische Hochschule Hannover; J. Fritze, Verband der privaten Krankenversicherung e. V., DGPPN; J. Gensichen, Allgemeinmedizin Universität Jena; T. Giese, Barmer-Ersatzkasse; U. Hapke, Robert KochInstitut; U. Hegerl, Psychiatrie Universität Leipzig; H. Irle, DRVBund; F. Jacobi Psychologie, TU Dresden; U. John, Epidemiologie und Sozialmedizin Universität Greifswald; T. Kallert, Park-Krankenhaus Leipzig; P. Kling-Lourenco, Psychiatrie Günzburg, Universität Ulm; H-H. König, Gesundheitsökonomie Universität Leipzig; H. Kunze, Aktion psychisch Kranke; M. Linden, Rehaklinikum Seefeld, Charité; C. Norra, Psychiatrie Universität Bochum; W. Reichwaldt, Firma Janssen-Cilag; C. Roick, AOK-Bundesverband; T. Ruprecht, Techniker Krankenkasse; M. Schützwohl, Psychiatrie und Psychotherapie TU Dresden; A. Statz, Bundesministerium für Gesundheit; T. Steinert, Psychiatrie Weissenau, Universität Ulm; U. Walter, Prävention Medizinische Hochschule Hannover.

\section{Literatur}

1 Arbeitskreis Versorgungsforschung beim Wissenschaftlichen Beirat der Bundesärztekammer. Definition und Abgrenzung der Versorgungsforschung. www.bundesaerztekammer.de 2004

2 Melchinger $H$, Rössler W, Machleidt W. Ausgaben in der psychiatrischen Versorgung. Ist die Verteilung der Ressourcen am Bedarf orientiert? Nervenarzt 2006; 77: 73-80

3 Claassen D, Priebe S. Deutschsprachige psychiatrische Versorgungsforschung - Was und wie wird berichtet? Eine Analyse von Veröffentlichungen in der Psychiatrischen Praxis 1999-2002. Psychiat Prax 2003; 30 (8): 414-423

4 Schneider N. Versorgungsforschung: Mehr Transparenz bei Interessenkonflikten. Dt Ärzteblatt 2008; 105: A943

5 Rossler W. Cui Bono oder wer braucht die Gesundheitsökonomie. Psychiat Prax 2001; 28 (Suppl. 1): S29-S31
6 Lomas J, Fulop N, Gagnon D et al. On being a good listener: Setting priorities for applied health services research. Milbank Q 2008; 81 (3): 363-388

7 Alonso J, Angermeyer M, Bernert S et al. Use of mental health services in Europe: results from the European Study of the Epidemiology of Mental Disorders (ESEMeD) project. Acta Psychiatr Scand 2004; 109 (Suppl. 420): 47-54

8 Alonso J, Codony $M$, Kovess $V$ et al. Population level of unmet need for mental healthcare in Europe. Br J Psychiatry 2007; 190: 299-306

9 Demyttenaere K, Bruffaerts R, Posada-Villa J et al. Prevalence, severity, and unmet need for treatment of mental disorders in the World Health Organization World Mental Health Surveys. JAMA 2004; 291 (21): 2581-2590

10 Cuijpers $P$, van Straten A, Smit F. Preventing the incidence of new cases of mental disorders. A meta-analytic review. J Nerv Ment Dis 2005; 193: $119-125$

11 Thomas $R$, Perera $R$. School-based programmes for preventing smoking. Cochrane Database Syst Rev 2006; (3): CD001293

12 Petermann $H$, Roth $M$. Suchtprävention im Jugendalter. Interventionstheoretische Grundlagen und entwicklungspsychologische Grundlagen. Weinheim: Juventa Verlag, 2006

13 Foxcroft $D$, Ireland $D$, Lister-Sharp $D$ et al. Longer-term primary prevention for alcohol misuse in young people: a systematic review. Addiction 2003; 98 (4): 397-411

14 Thomas R, Baker P, Lorenzetti $D$. Family-based programmes for preventing smoking by children and adolescents. Cochrane Database Syst Rev 2007; (1): CD004493

15 Loeber S, Mann K. Prävention bei Alkoholismus. Was wirkt? Psychiat Prax 2006; 33: 108-116

16 Melchinger H, Machleidt W, Rössler W. Psychiatrische Versorgung, Ausgaben auf dem Prüfstand. Dt Ärzteblatt 2003; 44: C 2227-C 2228

17 Wittchen $\mathrm{H}-\mathrm{U}$, Kessler R, Beesdo $\mathrm{K}$ et al. Generalized anxiety and depression in primary care: Prevalence, recognition, and management. J Clin Psychiatry 2002; 63: 24-34

18 Fernández A, Haro J, Martinez-Alonso $K$ et al. Treatment adequacy for anxiety and depressive disorders in six European countries. Br J Psychiatry 2007; 190: 172-173

19 Kovess-Masfety V, Alonso J, Brugha Tet al. Differences in lifetime use of services for mental health problems in six European countries. Psychiatr Serv 2007; 58 (2): 213-220

20 Günther OH, Friemel S, Bernert S et al. Die Krankheitslast von depressiven Erkrankungen in Deutschland. Psychiat Prax 2007; 34: 292-301

21 Lesperance F, Frasure-Smith N, Bourrassa M. Five-year risk of cardiac mortality in relation to initial severity and one-year changes in depression symptoms after myocardial infarction. Circulation 2002; 105: 1049-1053

22 Kubzansky L, Davidson K, Rozanski A. The clinical impact of negative psychological states: expanding the spectrum of risk for coronary artery disease. Psychosom Med 2005; 67 (Suppl. 1): 10-14

23 Grace S, Abbey SE, Kapral M et al. Effect of depression on five-year mortality after an acute coronar syndrome. Am J Cardiol 2005; 96: $1179-1185$

24 Sachverständigenrat für die Konzertierte Aktion im Gesundheitswesen. Depressive Störungen. In: Sachverständigenrat für die Konzertierte Aktion im Gesundheitswesen, editor. Gutachten 2000/2001: Bedarfsgerechtigkeit und Wirtschaftlichkeit, Ausführliche Zusammenfassung. 2001: 169-177

25 European Centre for Health Policy, WHO Regional Office for Europe. Health impact assessment. Main concepts and suggested approach. Gothemburg consensus paper. www.euro.who.int Brussels: 1999

26 Roick C, König H. Quo vadis? Gesundheitspolitische Entscheidungen und deren Konsequenzen für die Psychiatrie. Psychiat Prax 2005; 32: 109-112

27 Gandjour A, Lauterbach K. Wann lohnt sich der Abbau medizinischer Unter- und Überversorgung? Das Beispiel der Behandlung akuter Depressionen. Psychiat Prax 2004; 31 (3): 157-162

28 Bramesfeld A. Wie gemeindenah ist die psychiatrische Versorgung in der Bundesrepublik Deutschland? Psychiat Prax 2003; 30: 256-265

29 Albrecht D, Bramesfeld A. Das Angebot an gemeindenahen beruflichen Rehabilitationsmöglichkeiten für psychisch kranke Menschen in der Bundesrepublik. Gesundheitswesen 2004; 66: 492-498

30 Gutamann A, Thompson D. Why deliberative democracy? Princton: 2004

31 Salize H-J, Rössler W, Becker T. Mental Health Care in Germany. Eur Arch Psychiatry Clin Neurosci 2007; 257: 92-103 